> Dans les années 1980, le développement de la biologie moléculaire a permis de cloner rapidement des fragments d'anticorps recombinants sous forme de scFv (single chain Fv) fonctionnels. En raison de leur faible taille, et de leur relative facilité d'expression, les scFv présentent de nombreuses applications médicales et biotechnologiques. L'ADN codant ces anticorps peut ainsi être aisément exprimé dans des cellules de mammifères et les anticorps synthétisés ciblés vers différents compartiments cellulaires grâce à des séquences d'adressage permettant leur transport ou leur rétention dans un compartiment donné. L'anticorps recombinant peut ainsi être utilisé comme anticorps intracellulaire (intrabody) pour neutraliser, perturber ou suivre la dynamique endogène d'un antigène. Les intrabodies présentent non seulement une nouvelle voie pour la recherche fondamentale en permettant l'étude de la dynamique de protéines endogènes, mais aussi des perspectives intéressantes pour la recherche appliquée en terme d'immunisation intracellulaire en vue d'une utilisation thérapeutique. <
Utilisation des intrabodies : de l'étude des protéines intracellulaires à l'immunisation thérapeutique

Sandrine Moutel, Franck Perez

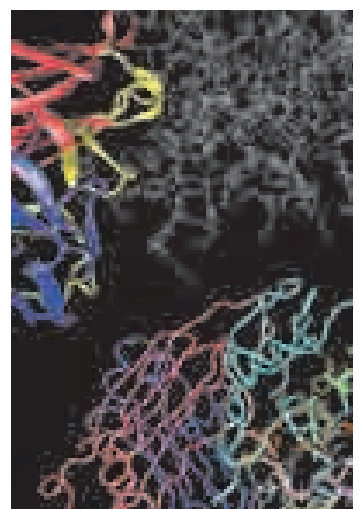

CNRS UMRI44,

Compartimentation et dynamique cellulaires, département de recherche translationnelle, Institut Curie, 26, rue d'Ulm, 75248 Paris Cedex 05, France. Franck.Perez@curie.fr
Les anticorps (Ac) polyclonaux ou monoclonaux (Acm) sont utilisés depuis de nombreuses années pour perturber la fonction et la dynamique de protéines intracellulaires [1]. Cette technique, puissante parce qu'elle permet d'inactiver ponctuellement la fonction des protéines ciblées, reste limitée par la nécessité de purifier et de concentrer les anticorps qui doivent donc être disponibles en grande quantité et résister à l'agrégation. Cette approche intracellulaire est en général basée sur la microinjection ce qui limite le nombre de cellules observables, bien que l'électroporation ou I'utilisation de réactifs de transfection aient aussi été utilisées avec succès.

Avec le développement de l'ingénierie génétique des Ac [2] $(\rightarrow)$, il a été possible de revisiter cette approche en utilisant des anticorps recombinants produits dans des cellules

$(\rightarrow)$ voir $R$. Abès et al., page 1011

après transfection de l'ADNc correspondant [3, 4]. Ces anticorps intracellulaires, ou intrabodies ont depuis été appliqués à l'étude et à la perturbation de l'activité de protéines cellulaires, et ce dans une optique soit de recherche fondamentale soit thérapeutique. Les étapes permettant le développement d'un intrabody doivent tenir compte de l'origine, naturelle ou synthétique, de l'anticorps, de ses caractéristiques de liaison à son antigène, de sa résistance aux conditions intracellulaires et de l'application souhaitée (Figure 1).

\section{Expression intracellulaire}

Le format scFv (fragments variables simple chaîne) est le plus couramment utilisé pour les intrabodies. II est constitué uniquement des domaines variables des chaînes lourdes et légères (L) VH et VL des anticorps, reliés entre eux par un lien peptidique flexible (généralement quinze acides aminés).

En sous-clonant ces fragments d'anticorps dans des vecteurs modifiés pour permettre une expression intracellulaire, il est ainsi possible d'inactiver certaines protéines, le fragment d'Ac se fixant directement à un domaine fonctionnel de ces dernières, perturbant l'interaction avec un partenaire ou encore retenant la protéine dans un compartiment cellulaire particulier. 


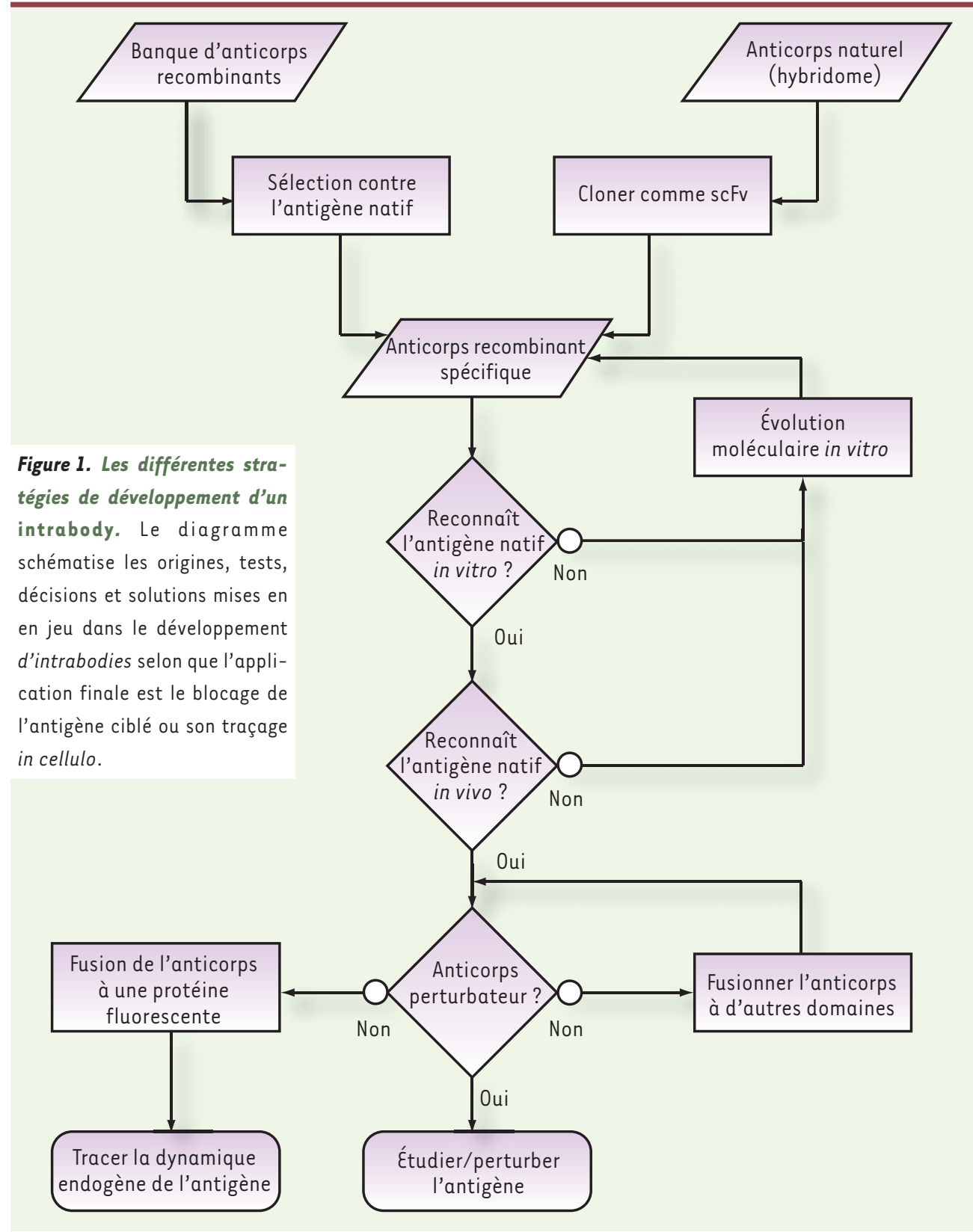

VL ou VH et les single domain VHH de camélidés [7] $(\rightarrow)$. Les $(\rightarrow)$ voir formats sim- P. Chames ple VL, simple et D. Baty, VH ou VHH page 1159 ont l'avantage d'être généralement solubles et stables en milieu réducteur car, étant des monomères, ils n'ont pas de ponts disulfures inter-chaînes. II a de plus récemment été montré que le manque de ponts disulfures intrachaîne n'est pas préjudiciable à la fonctionnalité du VH ou VL [8]. Comme dans le cas des scFv, les régions charpentes des VHH peuvent être encore améliorées pour mieux résister à la réduction [9].

\section{Applications à la recherche fondamentale}

Les intrabodies dont l'expression perturbe les protéines ciblées représentent bien sûr un outil de choix pour la recherche fondamentale. Cependant, tous les intrabodies ne sont pas bloquants et ils peuvent aussi se lier à une protéine sans en gêner sa fonction. Ces

Seuls les anticorps détectant la forme native de leur cible sont éligibles pour cette approche, mais ce n'est pas la seule limitation des intrabodies. Le repliement des scFv est défavorisé dans les conditions réductrices présentes dans le cytosol et nombreux sont les scFv qui sont incapables de reconnaitre leur cible dans ces conditions. Ils peuvent aussi précipiter et/ou être rapidement dégradés. De nombreuses approches ont été développées pour surmonter ces limitations comme l'évolution moléculaire ou l'utilisation de régions charpentes résistantes à la réduction $[5,6]$. Il est aussi possible d'effectuer la sélection de l'intrabody directement en milieu réducteur, par exemple en effectuant le criblage en système de levure double-hybride.

D'autres formats que les scFv ont aussi été utilisés avec succès : les Fab, les anticorps divalents ou bispécifiques (diabodies), les single domain anticorps peuvent cependant être manipulés, notamment en étant fusionnés à d'autres protéines ou à des signaux particuliers et ainsi devenir des perturbateurs spécifiques.

Par ailleurs, nous avons montré que les intrabodies non bloquants peuvent représenter des outils uniques pour suivre la dynamique des protéines endogènes sans que l'on ait recours à la surexpression de protéines sauvages ou mutées. La dynamique d'une très grosse protéine de l'appareil de Golgi, la giantine, a ainsi pu être étudiée grâce à un scFv anti-giantine étiqueté avec la GFP (green fluorescent protein) et exprimé in vivo [10]. Différents intrabodies fluorescents ont ainsi 


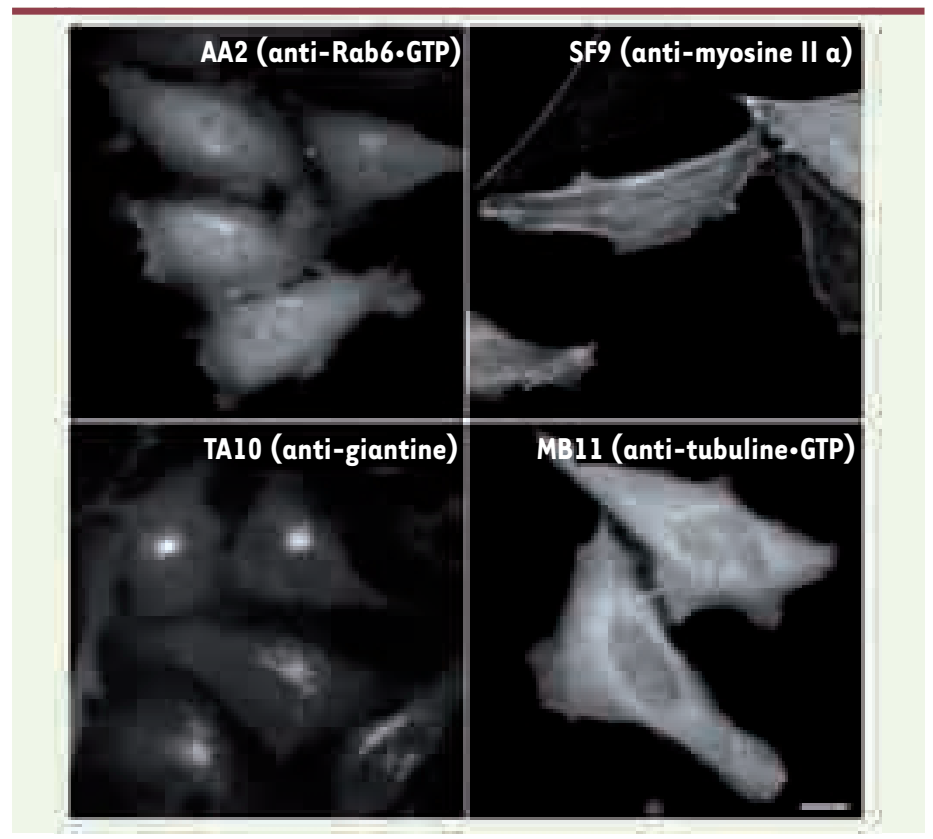

Figure 2. Quelques exemples d'intrabodies permettant l'analyse de la dynamique intracellulaire des protéines ciblées. Les anticorps scFv dirigés contre la forme liée au GTP de Rab6, de la myosine II, de la giantine ou de la tubuline liée au GTP ont été fusionnés à la GFP et exprimés dans des cellules HeLa. Après un délai de seize heures après l'expression des scFv, les cellules ont été observées vivantes et intactes par microscopie à fluorescence. La localisation dynamique des différentes protéines endogènes ciblées peut ainsi être analysée. Barre d'échelle : $10 \mu \mathrm{m}$.

été développés dans le laboratoire (quelques exemples sont présentés dans la Figure 2).

Dans d'autres études, nous avons montré que les méthodes de crible par la technique du phage display permettent de sélectionner des scFv dits conformationnels ne détectant que certains états tridimensionnels de la protéine ciblée. Nous avons ainsi réussi à produire des anticorps dirigés contre les formes liées au GTP de Rab6 [11] ou de la tubuline [12]. Ces anticorps utilisés comme intrabodies nous ont permis d'obtenir des résultats originaux: nous avons ainsi mis en évidence la dynamique endogène de cette protéine [11] et de l'anti-tubuline et avons proposé un nouveau modèle expliquant la dynamique instable des microtubules [12]. La sélection d'intrabodies a aussi été réalisée en utilisant des anticorps de lamas qui semblent montrer une meilleure résistance à la réduction cytoplasmique [13] $(\rightarrow)$.

$\rightarrow$ voir P. Chames et D. Baty, page 1159

\section{Applications thérapeutiques}

Les intrabodies représentent une approche attractive pour l'immunothérapie. Les scFv ont en effet démontré leur efficacité depuis une quinzaine d'années aussi bien dans le cas de maladies virales $(\rightarrow)$, que pour modifier le phénotype des cellules can-

$\rightarrow$ J.P. Sibilia, page 1033 céreuses. Nous illustrerons cette utilisation par deux exemples. En 1993, un intrabody reconnaissant le site de liaison de la gpl20 du virus de l'immunodéficience humaine (VIH-1) à la molécule CD4 a été utilisé comme agent thérapeutique bloquant ainsi la réplication du virus. Le scFv a été exprimé de façon stable et retenu dans le réticulum endoplasmique grâce à une séquence spécifique ( $\kappa D \varepsilon L)$ sans exercer de toxicité envers les cellules. En se fixant à la protéine virale d'enveloppe à l'intérieur de la cellule, il a conduit à une réduction substantielle du nombre des nouvelles particules virales produites [14]. En 1998, un autre intrabody dirigé contre la protéine Ras mutée a permis de bloquer la prolifération cellulaire, et d'induire l'apoptose dans des modèles d'oocytes de Xenopus laevis et dans des fibroblastes NIH3T3. Ce scFv a aussi induit des régressions tumorales lorsqu'il a été utilisé dans un modèle de souris immunodéprimées greffées avec une tumeur de côlon humaine. Dans ce cas, l'administration intracellulaire de l'intrabody avait été réalisée via l'injection intratumorale d'un adénovirus contenant l'ADNc codant pour l'anticorps [15].

Ces résultats soulignent l'intérêt thérapeutique potentiel des anticorps intracellulaires, notamment dans le traitement de certains cancers. Comme l'une des propriétés intrinsèques des intrabodies est la reconnaissance avec une haute spécificité de toutes les isoformes d'une protéine, incluant leur conformation pathologique, ces molécules suscitent à l'heure actuelle de nombreux espoirs pour le traitement de maladies résultant de l'accumulation de protéines mal repliées: maladie d'Alzheimer, de Parkinson, d'Huntington ainsi que l'encéphalopathie spongiforme liée au prion [16].

Malgré tout, l'absence de vecteurs sûrs et efficaces délivrant ces intrabodies reste un obstacle majeur à leur utilisation thérapeutique. Certes l'intrabody peut être aussi introduit à l'intérieur d'une cellule par des techniques alternatives, comme des vecteurs de type lipidique ou après leur fusion à des polypeptides permettant de franchir la membrane plasmique (protein transduction domain) (PTD). D'autres problèmes devront aussi être résolus: la production en masse des anticorps, la mâ̂trise de leur dégradation après expression intracellulaire et la nécessité de répéter les injections pour assurer l'efficacité du traitement [17].

\section{Pour conclure}

La puissance des anticorps recombinants intracellulaires en termes d'efficacité et de spécificité, alliée au vaste spectre de leurs applications potentielles, notamment pour perturber la fonction et suivre la dynamique des protéines in vivo et bloquer des protéines virales ou oncogéniques, laissent entrevoir pour les intrabodies des perspectives uniques que ce soit en recherche fondamentale ou en recherche appliquée. $\diamond$ 


\section{SUMMARY}

Intrabodies, potent tools to unravel the function

and dynamics of intracellular proteins

In the 1980s, progress in molecular biology enabled the manipulation and cloning of antibody fragments as functional scFv (single chain Fv). Because of their small size and relative ease of expression, scFv opened the road for new medical and biotechnological applications. scFvs can be easily expressed and targeted to different cellular compartments (cytosol, nucleus, endoplasmic reticulum, mitochondria, inner surface of the plasma membrane, etc.), using specific signals to target or retain them in a given compartment. Recombinant antibodies can thus be used as intracellular antibodies (intrabody) to neutralize, disrupt or track endogenous antigen. Intrabodies not only represent new tools for fundamental research to study the dynamics of endogenous proteins, but may also bring interesting options for applied research in terms of intracellular immunization for therapeutic use. $\diamond$

\section{REMERCIEMENTS}

Le travail du laboratoire est soutenu par le CNRS et par le département de recherche translationnelle et le centre de recherche de l'Institut Curie. Le travail portant sur les anticorps recombinants est en outre soutenu par HFSPO (human frontier science program).

\section{CONFLIT D'INTÉRÊTS}

Les auteurs déclarent n'avoir aucun conflit d'intérêts concernant les données publiées dans cet article.

\section{RéFÉRENCES}

1. Kreis TE. Microinjected antibodies against the cytoplasmic domain of vesicularstomatitis virus glycoprotein block its transport to the cell surface. EMBO J 1986; 5 : 931-41.

2. Orlandi R, Güssow DH, Jones PT, Winter G. Cloning immunoglobulin variable domains for expression by the polymerase chain reaction. Proc Natl Acad Sci USA 1989 ; 86 : 3833-7.

3. Biocca S, Neuberger MS, Cattaneo A. Expression and targeting of intracellular antibodies in mammalian cells. EMBO J $1990 ; 9$ : 101-8.
4. Biocca S, Ruberti F, Tafani M, et al. Redox state of single chain Fv fragments targeted to the endoplasmic reticulum, cytosol and mitochondria. Biotechnology (NY) $1995 ; 13$ : 1110-5.

5. Philibert $P$, Stoessel $A$, Wang $W$, et al. A focused antibody library for selecting scFvs expressed at high levels in the cytoplasm. BMC Biotechnol $2007 ; 7: 81$.

6. Visintin M, Settanni G, Maritan A, et al. The intracellular antibody capture technology (IACT) : towards a consensus sequence for intracellular antibodies. J Mol Biol $2002 ; 317$ : 73-83.

7. Holliger P, Hudson PJ. Engineered antibody fragments and the rise of single domains. Nat Biotechnol $2005 ; 23$ : 1126-36.

8. Tanaka T, Rabbitts TH. Functional intracellular antibody fragments do not require invariant intra-domain disulfide bonds. J Mol Biol 2008 ; $376: 749-57$.

9. Saerens D, Pellis M, Loris R, et al. Identification of a universal VHH framework to graft non-canonical antigen-binding loops of camel single-domain antibodies. J Mol Biol 2005; 352: 597-607.

10. Nizak C, Martin-Lluesma S, Moutel S, et al. Recombinant antibodies against subcellular fractions used to track endogenous Golgi protein dynamics in vivo. Traffic $2003 ; 4: 739-53$.

11. Nizak C, Monier $S$, del Nery $\varepsilon$, et al. Recombinant antibodies to the small GTPase Rab6 as conformation sensors. Science 2003 ; 300 : 984-7.

12. Dimitrov A, Quesnoit M, Moutel S, et al. Detection of GTP-tubulin conformation in vivo reveals a role for GTP remnants in microtubule rescues. Science 2008 ; 322 : 1353-6.

13. Rothbauer U, Zolghadr K, Tillib S, et al. Targeting and tracing antigens in live cells with fluorescent nanobodies. Nat Methods $2006 ; 3: 887-9$.

14. Marasco WA, Haseltine WA, Chen S. Design, intracellular expression, and activity of a human anti-human immunodeficiency virus type $1 \mathrm{gp} / 20$ single-chain antibody. Proc Natl Acad Sci USA 1993; 90 : 7889-93.

15. Cochet 0 , Kenigsberg M, Delumeau I, et al. Intracellular expression of an antibody fragment-neutralizing p21 Ras promotes tumor regression. Cancer Res $1998 ; 58: 1170-6$.

16. Cardinale A, Biocca S. The potential of intracellular antibodies for therapeutic targeting of protein-misfolding diseases. Trends Mol Med $2008 ; 14: 373-80$.

17. Lobato MN, Rabbitts TH. Intracellular antibodies and challenges facing their use as therapeutic agents. Trends Mol Med 2003; $9: 390-6$.
Tarifs d'abonnement M/S - 2010

Abonnez-vous

à Médecine/Sciences
$>$ Depuis 20 ans, grâce à $m / s$, vous vivez en direct les progrès des sciences biologiques et médicales

TIRÉS À PART

F. Perez 